\title{
The Dark Database: Facial Recognition and Its "Failure" to Enroll
}

\section{DENNIS DELGADO}

Adjunct Assistant Professor, State University of New York at Suffolk

\begin{abstract}
This essay discusses the Dark Database project, which looks at the presence of blackness in facial recognition systems. It recognizes the system's privileging of Caucasian skin as the central definition of skin tone. The essay considers these flaws within the context of photography's history of shaping how people of color are imagined and represented in visual culture. Moving through the structure of a facial recognition system, the essay describes utilizing that system to create a set of composite portraits that record what a system can "see," and furthermore, what that process can tell us about the overall culture that produced it.
\end{abstract}

\section{INTRODUCTION}

Over the course of 2019 and 2020 the Dark Database project crystallized, in the simplest of terms, into a set of composite portraits. Each portrait represents the median pixel value of all of the faces detected by an Open Computer Vision algorithm when executed on a feature-length film. Each detected face has been centered, enlarged, and layered to create a single composite portrait using a facial template as a guide. Because facial recognition systems privilege Caucasian skin tones as the range of values that constitute the definition of skin, they often do not detect and consequently enroll faces that contain darker skin. As a direct result of this, these systems have difficulty identifying people of color, because the databases they access lack diversity. The algorithm used to detect each face was run on a selection of films directed by African-American men. The films chosen were Training Day, ${ }^{1}$ Do the Right Thing, ${ }^{2}$ Higher Learning, ${ }^{3}$ and Black Panther. ${ }^{4}$ Using software to calculate the median pixel values in each portrait, a uniquely unrecognizable face emerged (see figure 1). What does this face mean? What does this face evoke? 


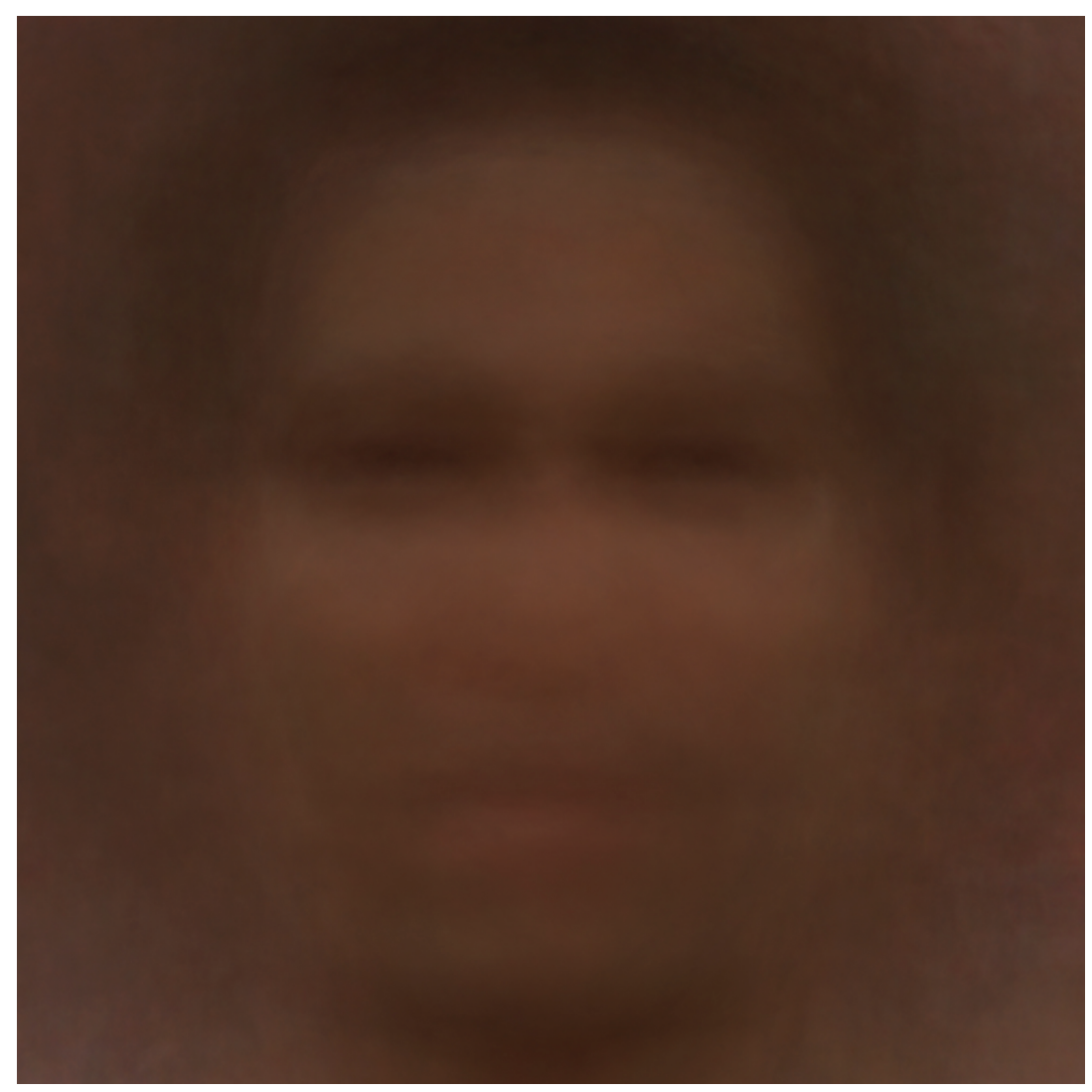

Figure. 1. Do The Right Thing, 2020 (C) Dennis Delgado.

In my research as an artist, one of the most controversial aspects of facial recognition is that it demonstrates bias with regard to people of color (among other social groups). I am interested in how photography and technologies of vision help to establish and maintain the construct of race, as well as how they bolster existing power structures. Facial recognition is a contemporary iteration of this racist tradition. As an artist I am interested in trying to find forms that encapsulate and expose these issues. What the Dark Database reveals is that facial recognition is yet another cultural form that shapes our imaginations, indirectly telling us what and whom to fear, and ultimately producing a kind of phantasm. What follows is a slightly nonlinear narrative of how the work took shape, what the work reveals, and how a previous project helped establish the foundation for the Dark Database.

In 2018 I looked at what other artists were saying about facial recognition, and what they were ultimately revealing or critiquing in their work. One of the first artists who interested me on the subject was Paolo Cirio, and his project Obscurity. Cirio's Obscurity project effectively disrupts the networks that decimate facial recognition data. The project hacked into a number of online 
databases containing mugshots and blurred the images as a protest against the violation of the right to privacy of individuals who had been arrested and photographed, and whose data was being made available online. ${ }^{5}$ The blurred mugshots had a ghostly quality to them that I found fascinating.

I had already been thinking about ghosts while making a film at the Bronx Zoo in 2013. I had been looking at how African men were treated in American society in the early twentieth century, beginning with the ethnographic display of Ota Benga in 1906 at the New York Zoological Society (now known as the Bronx Zoo). Ota Benga was an African tribesman brought to the United States to be exhibited originally at the World's Fair in St. Louis, in 1904. Benga had been chosen to serve as a living example of one of the steps of evolution from primates to human beings (or homo sapiens) when he was exhibited at the Bronx Zoo in September of 1906. Benga was meant to demonstrate how Africans were "primitive" beings, not as fully evolved or "civilized" as their European white male counterparts. He was put on display in the Primate House along with an orangutan in one of the house's outdoor cages. I was so upset by the story of Ota Benga that I felt compelled to make a film that looked at the site of what I considered to be a horrific crime. In doing research for the film, I discovered a number of texts that were instrumental in shaping my thinking on what it meant to try and say something about an incident that took place over a hundred years ago. Were there any traces of this crime today? I read the performance artist Coco Fusco's essay, The Other History of Intercultural Performance; one of the questions she and Guillermo Gomez-Pena proceeded from as they enacted a performance on ethnographic display was, "Had things changed, we wondered? How would we know, if not by unleashing those ghosts from a history that could be said to be ours?"6 It was this idea of revealing "ghosts," that I wanted to undertake myself.

At around the same time, I had read T.J. Demos's book Return to the Post-Colony: Specters of Colonialism in Contemporary Art. This also helped frame my area of inquiry as I tried to find a physical, or to use Demos's language, a "spectral” trace of a past colonial crime. Demos's use of Derrida's Hauntology concept captivated me, and I think that is what initially caught my eye in Paolo Cirio's work (mentioned above) — an image of a face that is blurry and therefore difficult to read or discern an identity from, and which looks something like a ghost.

I therefore began to think about the film I was making as a kind of search for ghosts. I believe the Dark Database project continues this investigation. When I returned to Demos's text again recently, one particular quote resonated:

Again, the problem is not one of dealing with spirits from another world; rather, it's a matter of being sensitive to modernity's phantoms - that is, the disturbances and lingering presences, or presences of absence in the orders of visual appearance, through which current social formations manifest the symptomatic traces and uncanny signs of modernity's history of violence and exclusions. ${ }^{7}$

This quote has helped me to understand how the portraits of the Dark Database can serve to reveal an absence and consequently an exclusion — an epistemic violence — that had begun in many ways over a hundred years ago. When I finally finished my project on Ota Benga, I ended up with a large number of photographs, as well as three different videos which looked at the Ota Benga incident and the current practices of the Wildlife Conservation Society. This project helped 
me to understand that I had an interest in technologies of vision and their relationship to colonialism, race, and surveillance. A quote from Catherine Russell's book Experimental Ethnography brought these interests into sharp focus:

As a form of representation, the zoo is a technology of vision; the zoological gaze is an apparatus that is also a cultural practice in which the Other (species) is brought close and yet kept apart, at a safe distance. The zoo as a "discipline" in Foucault's sense is a form of knowledge that is produced in and through technologies of vision. ${ }^{8}$

What knowledge is being produced within the confines of facial recognition systems? Does the early twentieth-century technology of the zoo cage find its echo in the twenty-first century technology of artificial intelligence? Looking at the images in the Dark Database, it appears so. As Kelly A. Gates reflects on facial recognition systems in her book Our Biometric Future: Facial Recognition Technology and the Culture of Surveillance:

Taken together, they present a more complete picture of the ways in which automated forms of identification over networks serve the neoliberal aims of mass individuation and social differentiation, a means of tying individuals into circuits of inclusion and exclusion, determining their status as legitimate citizens or more problematic identities. ${ }^{9}$

In both the zoo cage and the facial recognition system, a separation is imposed and a false hierarchy created, and consequently, a false knowledge is produced. Indeed, one of the most memorable moments of creating the Dark Database was when the facial detection algorithm began to find faces in the purely textual black-and-white title sequence of John Singleton's film Higher Learning. I realized that the composite portrait was not only going to reveal omissions, but also contain errors and fragments that were not faces. This meant that the cumulative knowledge being presented was only partial and erroneous at best. When the first two portraits of the Dark Database were completed, I realized I had finally found my ghosts.

Another text that found its way into my research early on was Mary Shelley's Frankenstein. I originally read the text when I was editing one of the videos on Ota Benga and decided to use passages from it to narrate parts of the film. At the time it was an experiment, and I did not know why I felt the need to include the text. However, I found some ideas later on that suggested my unconscious was making connections that were not immediately obvious to me. At the same time, I continued to look at other artists who were critiquing artificial intelligence, and I realized that none of the artists I could find were dealing with the issue of race or skin tone. That fact made me determined to add additional portraits that would speak to this issue. Ultimately, the Dark Database, as a whole, is a collection of records of what I think of as colonial ghosts. It is both a record of what an open-source facial detection algorithm can "see," and also what it can miss, even in films where the main characters are almost exclusively African-American, and where that protagonist's graphic weight is at its greatest. Could the collision of an African-American director's vision with the vision of artificial intelligence conjure up a specter? Again, with the Dark Database images, it appears so. It was at this time that an opportunity presented itself for me to showcase some of the work in the Dark Database.

I was invited to participate in an exhibition called The Black Index. The exhibition looked at how African-American artists were challenging the indexical relationship between photography and race, ultimately presenting a much more imaginative and alternative understanding of how people 
of color are portrayed in the landscape of visual culture. I proposed the first two portraits that currently make up part of the Dark Database project. I presented the images on Apple iPad Pros, mounted to the wall in enclosures, as a way to refer back to the technology we have all come to use on a daily basis, i.e., mobile devices. The hardware contains both the optics, software, and glass that not only allow for viewing the image, but that also cast one's own reflection on the surface of the screen.

It was one event in particular that made me decide that the images should be presented on the same technology that incorporated the flawed algorithms. In September of 2019, a friend and I went to see the painter Amy Sherald's exhibition, the heart of the matter... at the Hauser and Wirth gallery in New York City. Sherald is primarily known as the artist who painted the portrait of First Lady Michelle Obama. My friend and I casually stopped to take a picture of one of the artist's paintings. The painting, called Precious Jewels by the Sea, depicts two young AfricanAmerican men dressed in swim trunks standing on a beach, with two young African-American women sitting on their shoulders. As my friend raised her iPhone to take the picture, I saw the familiar rounded rectangles indicating where the camera detected faces, in this case within the painting. The bottom two rectangles, surrounding the two men's faces, were blinking on and off, and I immediately pointed out to my friend that the two men holding the African-American women had darker skin, and so the facial recognition algorithm on her iPhone was having trouble detecting the two African-American men's faces. My friend's jaw dropped as we experienced for the first time what I had been reading about, and what I had also been telling her about — my project utilizing facial recognition (see Figure 2).

While I realize that this example is a rather benign one, facial recognition systems and the flaws they contain can have real, and potentially disastrous consequences on people's lives, particularly when agencies such as law enforcement and homeland security increasingly rely on these systems. 


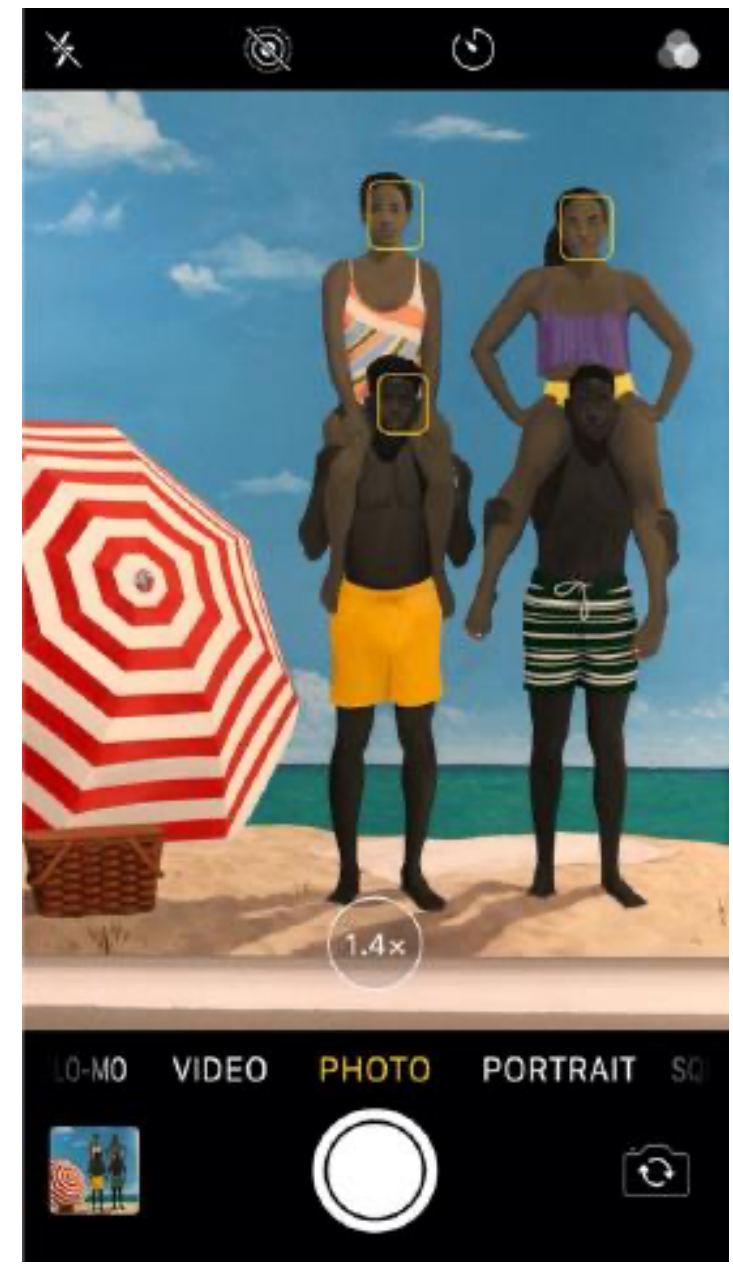

\section{Figure. 2. Photo of Precious Jewels by the Sea, 2019, Dennis Delgado, .jpeg (C) Dennis Delgado.}

It is important to understand exactly how facial recognition systems work. Facial recognition systems must be trained by quantifying portraits of people from which they create measured "facial templates." They use these templates to compare and possibly match an identity with a newly presented image of an unidentified person. What is being quantified depends on the system; however, all of them measure distances between predetermined points on a face. The points often fall on distinctive features in the face, for example the width at the base of the nose or the distance between the eyes. Points are mapped onto the detected face, and the distance of those points, as well as the three-dimensional shading that is read in numbers (corresponding to pixel values), all create a mathematical model of the detected face. Once those calculations are complete, then that model (or facial template) is compared to stored models which are numerically close in value. As Christy Lange, journalist and leader of Tactical Tech summarizes in an article on facial recognition, "Once the software detects a face, it compares it to a set of others to find the best statistical 'match,' a calculation that is always relative and, therefore, can never be 100 percent accurate." 10 The "set of others" [other faces] forms what is an important 
component of facial recognition's accuracy. That is to say that the database of faces that the software has access to determines if it will recognize a face accurately as one that it has measured or "seen" before.

As the number of facial templates that constitute a single-identity increases, the neural network comparing these templates makes adjustments to each model with the goal of accumulating as much information as possible that points to a single identity. In other words, the more images it has of a person, the more it can accurately identify that person. One of the most controversial aspects of this is that people of color - or more accurately, people who have darker skin than most Caucasian people - are often not "enrolled" into these databases because their faces cannot be detected in a photograph or from a live surveillance camera feed. In other words, the red, green, and blue values that are contained in the detection algorithm correspond to Caucasian skin. The range of values does not contain numbers that correspond to darker skin types. The camera, and the computer algorithms integrated into it, struggle to recognize darker-skinned faces as a result. As Simone Browne, Associate Professor of African and African-American Diaspora Studies at the University of Texas, points out in her book Dark Matters: On the Surveillance of Blackness:

[...] facial scan technologies may produce higher failure to enroll rates for "dark-skinned users," not due to lack of distinctive features, of course, but to the quality of images provided to the facial scan system by video cameras optimized for lighter skinned users. What their research and development tells us is that their technology privileges whiteness, or at least lightness, in its use of lighting and in the ways in which certain bodies are lit and measured in the enrollment process. ${ }^{11}$

By failing to recognize and enroll a sector of the population, facial recognition is recreating social divisions and fixing the identities of a predetermined few. A dramatic example of this is the recent story of Robert Julian-Borchak Williams, who was incorrectly identified by a facial recognition system. The Detroit Police Department arrested Mr. Williams for a crime he had nothing to do with. Mr. Williams is African-American, and the Detroit Police Department ultimately admitted they (or, more accurately, the facial recognition system they were utilizing) had made a mistake. Mr. Williams was eventually released from police custody, but the initial confrontation and arrest could have ended differently had Mr. Williams resisted in any way. ${ }^{12}$ Mr. Williams or a member of his family could have been shot and possibly killed simply because a piece of software was not properly developed. One of the meanings of the word "recognition" that is pertinent here is "acknowledgement of the existence, validity, or legality of something." 13 Regarding a sector of the population as invalid or illegal immediately places them at risk, and this is essentially what happened to Robert Williams. As Browne describes, "racializing surveillance" occurs "when enactments of surveillance reify boundaries along racial lines, thereby reifying race, and where the outcome of this is often discriminatory and violent treatment."14

Bias is well documented in the research and development of facial recognition; what is less obvious is what this bias means. It is worth stating that being recognized by a facial recognition system does not mean that people of color will escape violence or discrimination. It is in many ways a "double bind" and the Dark Database offers no simple solution to this issue. While being accurately identified is important for establishing legality, for example, it does still present other challenges with respect to internalized racism. The attempts to automate the labor of surveillance 
are also automating the racism that remains so present in American life. If the Dark Database helps to reiterate how social pathologies transform and persist, then at the very least it can serve as a step toward self-reflection.

For all of artificial intelligence's claims of "objectivity," and numeric precision, it is clear that certain wavelengths of light are prioritized in the development of this technology. In other words, the technology inherits the limited and discriminatory ways that the culture that produced it maintains. In addition to abstracting and coding bias, what else does facial recognition technology produce? As Kelly A. Gates pinpoints, "technologies are thoroughly cultural forms from the outset, embodying the hopes, dreams, desires and especially the power relations and ideological conflicts of the societies that produce them." 15 Looking at the Dark Database it appears that the cultural form also embodies a society's nightmares. What I think the Dark Database project points to is a continued fear of the Other, or (at the very least) a continued fear of the young Black male. The Dark Database brings into visibility that nightmare, in the form of a portrait. The more I sit with the project, the more I begin to imagine a database of unenrolled faces, too dark or too aberrant to be detected or solidly identified. In the same way that the technology of photography was used in the late nineteenth and early twentieth centuries to shape and delimit the social body (and to help define the features of the criminal face), facial recognition systems are classifying undetected faces as invisible, alien, or unknown, and potentially as a threat. Allan Sekula detailed this ordering process in his classic essay, "The Body and the Archive." Describing how composite mugshots and the taxonomic disciplines of phrenology and physiognomy were combined to create an all-encompassing and yet discriminatory archive, Sekula states:

We can speak then of a generalized, inclusive archive, a shadow archive that encompasses an entire social terrain while positioning individuals within that terrain. This archive contains subordinate, territorialized archives: archives whose semantic interdependence is normally obscured by the "coherence" and "mutual exclusivity" of the social groups registered within each. The general, allinclusive archive necessarily contains both the traces of the visible bodies of heroes, leaders, moral exemplars, celebrities, and those of the poor, the diseased, the insane, the criminal, the nonwhite, the female, and all other embodiments of the unworthy. ${ }^{16}$

Facial recognition systems are performing the same role in reverse: it is predominately the Caucasian face that is accurately identified. Further, the presence of blackness in facial recognition systems is a space that also allows for the society to project onto it a shared fear of the Other. As Toni Morrison states in her book Playing in the Dark (an analysis of the presence of blackness in the canon of Western literature):

For in the construction of Blackness and enslavement could be found not only the not free but also with the dramatic polarity created by skin color, the projection of the notme. The result was a playground for the imagination. ${ }^{17}$

This is what my project is helping me understand - this zone or space within facial recognition systems that contains a kind of monster. A monster that is all the more terrifying because of its invisibility and its disembodied and spectral trace. It is at the same time a promethean creation that traces its roots back to the monster in Mary Shelley's classic novel. The formation of 
"problematic identities," helps to shape the dominant imaginary in a very specific way, creating a specter that is both haunting and which serves as an unidentifiable, but undeniable presence (to invoke Toni Morrison's idea). What does this mystery face look like? How did it take shape? The theory of faciality developed by Deleuze and Guattari helps to understand how that face originally took shape.

Gates quotes Deleuze and Guattari as they write about the face of the Other and its ontological formation. Deleuze and Guattari trace this origin to the "white man," specifically Jesus. It is a discursive formation that in Deleuze's terms is created by an "abstract machine of faciality." Facial recognition mirrors this abstract machine, and processing images from films is one way to see how this machine creates a specter. It is worth quoting Deleuze and Guattari here at length:

The face that is produced by the abstract machine of faciality is an inhuman face, and facialization can be a violent process, transforming the radical and deeply relational potential of becoming into a dichotomous relationship between signifier and signified. The paradigmatic instance of facialization is the close-up up of the face in cinema, which destroys what is recognizable, social, and relational about the face, and "turns the face into a phantom."'18

This is another example of how the abstraction and omission of people of color forms a person we cannot recognize and which in effect haunts us. It is this phantom (or spirit) described by Deleuze and Guattari that is captured in the Dark Database. Mimicking eugenicist Francis Galton's use of photographic composites in the nineteenth century, I began assembling these composite images, some containing as many as six hundred faces from a single film. And indeed, a phantom is what I seem to be left with (see figures 2 and 3). As many viewers have pointed out, the images of the Dark Database also resemble religious icons and imagery. This ghost-like image is a kind of data visualization of what facial recognition systems can "see." What they cannot "see" is what is shaping a complementary un-enrolled and darker database. 


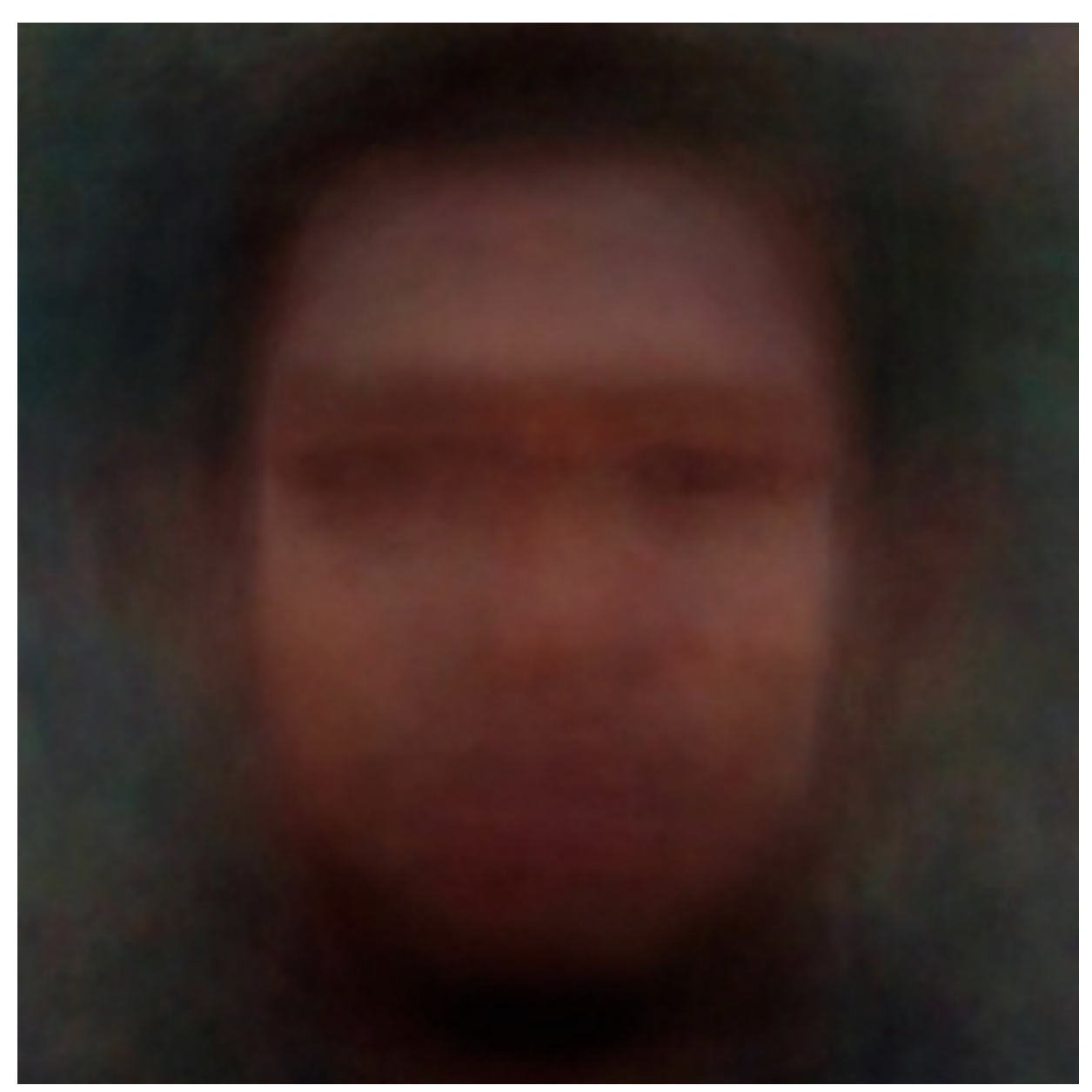

Figure 3. Black Panther, 2020, Dennis Delgado, Tagged Image File @ Dennis Delgado.

\section{ENDNOTES}

1. Training Day, directed by Antoine Fuqua (Warner Brothers, 2001).

2. Do the Right Thing, directed by Spike Lee (Universal Pictures, 1989).

3. Higher Learning, directed by John Singleton (Columbia Pictures, 1995).

4. Black Panther, directed by Ryan Coogler (Walt Disney Pictures, 2018).

5. Paolo Cirio, Obscurity, https://paolocirio.net/work/obscurity/ (accessed June 4, 2021).

6. Coco Fusco, "The Other History of Intercultural Performance," The Drama Review, Vol. 38, No. 1 (Spring, 1994), 143.

7. T.J. Demos, Return to the Postcolony: Specters of Colonialism in Contemporary Art (Sternberg Press, 2013), 13.

8. Catherine Russell, Experimental Ethnography: The Work of Film in the Age of Video (Duke University Press, 1999), 123.

9. Kelly A. Gates, Our Biometric Future: Facial Recognition and the Culture of Surveillance (New York University Press, 2011), 57. 
10. Christy Lange, "Surveillance, Bias and Control in the Age of Facial Recognition Software," https://frieze.com/article/surveillance-bias-and-control-age-facial-recognitionsoftware?language $=$ de $($ accessed October 29, 2019).

11. Simone Browne, Dark Matters: On the Surveillance of Blackness (Duke University Press, 2015), 113.

12. Kashmir Hill, "Wrongfully Accused by an Algorithm," https://www.nytimes.com/2020/06/24/technology/facial-recognition-arrest.html (accessed August 5, 2020).

13. Angus Stevenson, Oxford English Dictionary (Oxford University Press, 2010).

14. Simone Brown. Dark Matters: On the Surveillance of Blackness. (Duke University Press, 2015), 8.

15. Gates, Our Biometric Future, 12.

16. Allan Sekula, "The Body and the Archive," October, vol. 39 (Winter, 1986), 10.

17. Toni Morrison, Playing in the Dark (First Vantage Books, 1992), 38.

18. Gates, Our Biometric Future, 12.

\section{AUTHOR BIO}

Dennis Delgado was born in the South Bronx, and received a BA in Film Studies from the University of Rochester as well as an MFA in Sculpture from the City College of New York (CUNY). His work examines the forms through which ideologies of colonialism persist and reinscribe themselves, revealing a historical presence in the current moment. He is interested in how technologies of vision reproduce the scopic regimes of expansionism and neo-liberal governance. His work has been exhibited at the Bronx Museum of the Arts, the Schomburg Center for Research in Black Culture, El Museo del Barrio, and at the Cooper Union. 